\title{
LAS FORTALEZAS DE LOS MEXICANOS, UN ANÁLISIS DESDE LA AUTOPERCEPCIÓN
}

\author{
NORMA ALICIA RUVALCABA ROMERO*, JULIA GALLEGOS GUAJARDO**, ADRIANA MORALES SÁNCHEZ*** \\ UNIVERSIDAD DE GUADALAJARA, MÉXICO - UNIVERSIDAD DE MONTERREY, MÉXICO
}

Recibido: 29 de Marzo de 2016

Aprobado: 12 de Julio de 2016

\author{
Para referenciar este artículo: \\ Ruvalcaba Romero, N., Gallegos Guajardo, J. \& Morales Sánchez, A. (2016) Las fortalezas de los Mexicanos, un análisis desde \\ la autopercepción. Revista Iberoamericana de Psicología: Ciencia y Tecnología, 9 (1), 73- 84
}

\begin{abstract}
Resumen
El estudio de las virtudes y fortalezas humanas desarrollado en el marco de la Psicología positiva por Peterson y Seligman (2004), ha servido para la identificación de aspectos que puedan servir de contrapeso a los factores de riesgo que pueden afectar el bienestar de las personas. Objetivo: Este estudio busca identificar cuáles son las fortalezas prevalecientes en una muestra de personas mexicanas e identificar su posible concordancia con modelos previos que han caracterizado al mexicano. Método: Para este trabajo se aplicó el Inventario de Fortalezas y Virtudes (IVYF, Cosentino y Castro, 2008), así como un cuestionario de características sociodemográficas a 608 personas residentes en la Zona Metropolitana de Guadalajara. Resultados: Las fortalezas más auto-percibidas en los mexicanos son la integridad, la bondad, la gratitud, la perspectiva y la imparcialidad. Por otro lado, las menos percibidas son la autoregulación, el amor por el aprendizaje, la modestia, la ciudadanía y el humor. Asimismo se encontraron diferencias de género en 9 de las 24 fortalezas evaluadas, en las que la mujer tiende a tener puntuaciones más altas. Los resultados se discuten a través del contraste con estudios similares en otros grupos culturales y su concordancia con modelos previos de identificación de las cualidades de la población en México.

Palabras clave: Psicología positiva, fortalezas, virtudes, transcultural
\end{abstract}

\section{THE CHARACTER STRENGTHS OF MEXICANS, AN ANALYSIS FROM SELF-PERCEPTION}

\begin{abstract}
The study of virtues and character strengths developed under a positive psychology framework by Peterson \& Seligman (2004), has served to identify the positive traits that could help individuals to bounce back when experiencing risk factors that could otherwise affect their well-being. Aim: The present study aims to identify the most prevalent strengths within a sample of Mexican people, and their possible concordance with previous models that have characterized the Mexican. Method: Participants from this study completed the Inventario de Fortalezas y Virtudes (Inventory of Strengths and Virtues) (IFyV; Castro y Consentino, 2008) and a sociodemographic questionnaire. The participants were 608 people residents from the Metropolitan Zone of Guadalajara. Results: The most prevalent self-perceived strengths were: integrity, kindness, gratitude, perspective and fairness. On the other hand, the least prevalent self-perceived strengths were: self-regulation, love/eagerness to learn, modesty, civility and humor. Gender differences were also found on 9 of the 24 evaluated strengths, in which women reported higher scores than men. Results are discussed by contrasting current results with previous findings of studies carried out with other cultural groups, and also analyzing their accordance with the previous models that identified the positive traits of the Mexican population.

Keywords: Positive psychology, character strengths, virtues, cross-cultural
\end{abstract}

1 La correspondencia en relación a este artículo debe de ser enviada a: Norma Alicia Ruvalcaba Romero, Ph.D., Centro Universitario de Ciencias de la Salud, Universidad de Guadalajara, Sierra Nevada \#950, Guadalajara, Jalisco, C.P. 44290. México. Contacto: nruval@hotmail.com

* Ph.D. Centro Universitario de Ciencias de la Salud, Universidad de Guadalajara

** Ph.D. Centro Universitario de Ciencias de la Salud, Universidad de Guadalajara

*** Ph.D., Departamento de Psicología, Universidad de Monterrey. 


\section{La clasificación de las virtudes y fortalezas del carácter}

Para cualquier sociedad, el buen carácter es esencial para que sus individuos y comunidades florezcan (Martínez-Marti, 2006; Park, Peterson \& Seligman, 2006). Este está formado por virtudes y fortalezas. Las virtudes son las características centrales valoradas por tradiciones filosóficas y religiosas; las fortalezas, en un nivel más concreto, están asociadas a cada una de esas virtudes, proponiendo así un camino para desarrollarlas (Cosentino, 2009; Peterson \& Seligman, 2004).

El interés en el estudio de las fortalezas de carácter se ha desarrollado principalmente al interior de la rama de la psicología positiva, encontrando diferentes hallazgos que van desde su medición hasta sus características, prevalencias y efectos positivos en diversas áreas del ser humano (i.e., Castro, 2011; Corral-Verdugo, Tapia-Follem, Ortiz-Váldez, \& Frago-Sing, 2013; Martínez-Marti, 2006; Park, Peterson \& Seligman, 2006).

Partiendo de lo anterior, Peterson y Seligman (2004) proponen una clasificación del carácter moral. La clasificación sugiere seis virtudes que comprenden 24 fortalezas. La definición de cada fortaleza de carácter implica el cumplimiento de doce criterios básicos; según esta clasificación una fortaleza: debe ser universal; debe contribuir a la satisfacción; felicidad y plenitud humana; debe ser susceptible de ser moralmente valorada; no implica desvalorizar a otros; debe tener antónimos negativos; debe poseer cierto grado de generalización y estabilidad; debe ser medibles; no debe ser conceptual o empíricamente redundantes con otra fortalezas de carácter; debe ser un rasgo positivo intrínseco del individuo; su existencia se considera como prodigiosa al florecer en las etapas tempranas de niños y adolescentes; se encuentran ausentes en algunos individuos y, finalmente, existen instituciones que promueven de manera explícita prácticas dirigidas a cultivarlas (Park, Peterson, \& Seligman, 2006).

De acuerdo con Peterson \& Seligman, algunas de las fortalezas humanas más reconocidas son: Sabiduría y Conocimiento, que se refieren al aspecto cognitivo que facilita la adquisición y el uso del conocimiento, e incluye las fortalezas de creatividad, curiosidad, apertura mental, amor por el saber y perspectiva. La virtud del Coraje, que se refiere al aspecto emocional que involucra el ejercicio de la voluntad para alcanzar metas que afrontan adversidad, interna o externa. El coraje comprende las fortalezas de valentía, perseverancia, vitalidad e integridad. Las habilidades interpersonales involucradas en el entendimiento y en la amistad se encuentran dentro de la virtud de la Humanidad, que comprende las fortalezas de bondad, inteligencia social y amor. La virtud de la Justicia refiere al aspecto cívico, base de una comunidad saludable; allí figuran las fortalezas de imparcialidad, liderazgo y trabajo en equipo. La Templanza es la virtud que te protege ante el exceso, y en ella se encuentran autorregulación, prudencia, clemencia y modestia. La virtud de la Trascendencia proporciona un significado de vida y forja conexiones con un universo mayor, comprendiendo las fortalezas de la apreciación de la belleza y excelencia, gratitud, esperanza, humor y espiritualidad (Peterson \& Seligman, 2004). La descripción de cada fortaleza se encuentra en el anexo 1.

\section{Prevalencia y diferencias de género en las fortalezas de carácter}

Varios autores han investigado la prevalencia de las fortalezas de carácter en diferentes países. Park, Peterson \& Seligman (2006) realizaron un estudio con participantes de 54 naciones, que se aplicó a través del internet: Values in Action Inventory of Strenghts (VIA-S; Peterson \& Park, 2005). El estudio contó con 117.676 participantes. Se encontró que en Estados Unidos, las 5 fortalezas prevalentes fueron: amabilidad, justicia, integridad, gratitud y perspectiva, mientras que las menos prevalentes fueron: religiosidad, vitalidad, prudencia, modestia y auto regulación. En la población mexicana participante en el estudio $(\mathrm{N}=88)$ las 5 fortalezas prevalentes fueron: justicia, curiosidad, perspectiva, amor y gratitud; las menos prevalentes fueron: auto regulación, modestia, prudencia, vitalidad y persistencia. Al analizar los resultados de las 54 naciones en conjunto, los autores explican que en su mayoría, las personas alrededor del mundo se ven a sí mismas con altos puntajes de fortalezas interpersonales, pero con bajos niveles de templanza.

Con una muestra de 1.060 participantes de diferentes lugares de España, Azañedo, Fernández-Abascal \& Barraca (2014) encontraron que las 5 fortalezas prevalentes fueron: amabilidad, justicia, amor, gratitud y honestidad; mientras que perspectiva, prudencia, vitalidad, auto regulación y espiritualidad fueron las 5 menos prevalentes. Este estudio observó que las mujeres puntuaron más que los hombres en las fortalezas de: bondad, amor y gratitud; mientras que estos puntuaron más en creatividad.

Al igual que Park, Peterson, \& Seligman (2006), Linley, Maltby, Wood, Joseph, Harrington, Peterson, \& Park (2007) aplicaron el VIA-S por internet, con una muestra de 17.056 participantes del Reino Unido. Los resultados del estudio muestran que para las mujeres las 5 fortalezas con mayor prevalencia fueron: justicia, amabilidad, apertura mental, curiosidad y amor por el conocimiento; las 5 menos prevalentes en esta población fueron: esperanza, prudencia, 
modestia, auto regulación y espiritualidad. Para el género masculino las 5 fortalezas prevalentes fueron: apertura mental, justicia, curiosidad, amor por el conocimiento, y creatividad, mientras que las menos prevalentes fueron: esperanza, prudencia, auto regulación, modestia, y espiritualidad.

Los resultados de diferentes estudios muestran que en diversas culturas las fortalezas prevalentes corresponden a aquellas identificadas como valores universales y mínimos necesarios para una sociedad viable: acciones positivas de reciprocidad y cuidado del otro, penalidades en contra de la decepción y la traición, y normas de justicia en caso de conflicto (Bok, 1995; Park, Peterson, \& Seligman, 2006).

\section{Características culturales de los mexicanos}

El enfoque de las Dimensiones Culturales de Hofstede (2010), define la cultura como una programación colectiva de la mente que distingue a los miembros de un grupo o categoría de otros; consiste en las reglas no escritas en la interacción social. En este enfoque se identifican 5 categorías básicas por las que puede ser caracterizado un grupo cultural: Distancia o cercanía con el poder, individualismo o colectivismo, Masculinidad o feminidad, Evitación o tolerancia de la incertidumbre, Orientación a largo o a corto plazo, y Complacencia o Restricción.

Según los análisis del autor (Hofstede, Hofstede \& Minkov, 2010), en un comparativo entre 76 países con rango de respuesta de 0 a 100, las características culturales de los mexicanos que se lograron identificar fueron: Alto distanciamiento del poder; las personas aceptan un orden jerárquico sin cuestionamientos, el poder está centralizado, los subordinados esperan a que se les diga qué hacer. Asimismo su nivel de individualismo es bajo, lo que implica que la autoimagen se define en términos del grupo; la lealtad y el cuidado hacia los demás es primordial y se fomentan relaciones interpersonales sólidas entre los miembros del colectivo. La dimensión de masculinidad también se ubicó por encima de la media: en México prevalecen la competencia, el rendimiento y la pelea como estrategias para la resolución de los conflictos. Respecto a la evitación de la incertidumbre, México obtuvo altas puntuaciones, esto se refiere a la dificultad para aceptar situaciones no estructuradas o novedosas. Las personas que pertenecen a este tipo de cultura tienden a ser más emocionales, dogmáticas, intolerantes a creencias distintas, y suelen priorizar la seguridad como elemento importante en la motivación. La cultura mexicana también presentó puntajes muy bajos en lo que se refiere a la orientación a largo plazo, indicando un gran respeto por las tradiciones, escasa propensión al ahorro y la previsión, y una tendencia a obtener resultados rápidos. Finalmente, en la dimensión de complacencia se obtuvo la puntuación más alta: esto se relaciona con la voluntad de satisfacer sus impulsos, de divertirse y disfrutar la vida; tienden al optimismo, otorgan gran importancia al tiempo de ocio y utilizan su dinero para fines placenteros.

En la figura 1, se muestra un comparativo entre las puntuaciones de las dimensiones nacionales bajo el modelo de Hofstede, para México, España y Reino Unido, considerando que son los países de donde se obtuvieron los datos de los estudios comparativos del proyecto VIA (Azañedo, et al., 2014; Park et al., 2006).

Fig. 1. Comparativo de las dimensiones nacionales bajo el modelo de Hofstede entre México, Reino Unido y España.

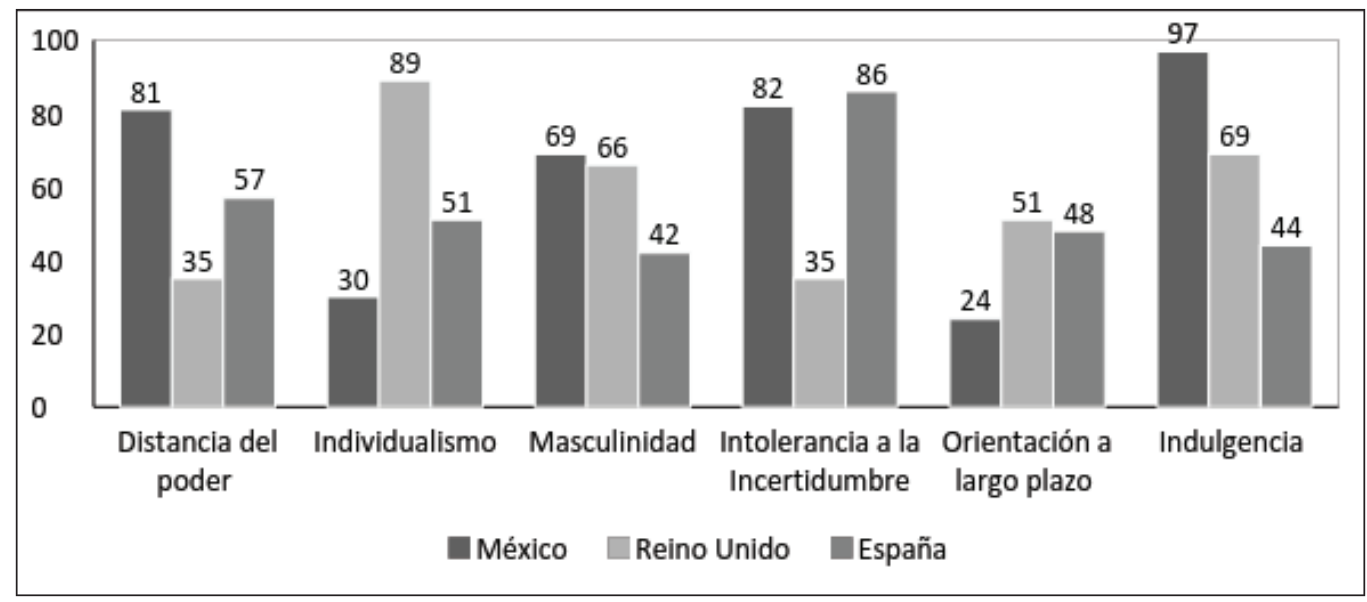

Nota: fuente adaptada http://geert-hofstede.com/mexico.html 
En lo que se refiere a los estudios nacionales, Díaz-Loving \& Draguns (1999), consideran que la cultura tiene 4 atributos esenciales: a) la concepción de sí mismo, b) la relación con la autoridad, c) el enfrentamiento al conflicto y d) el control de emociones.

Las culturas latinoamericanas se caracterizan por ser más colectivas que individualistas, su conducta está regida por mayor aproximación física y gestualidad (Díaz-Loving, 2005). La cultura mexicana es principalmente colectivista y subraya la importancia de las relaciones interpersonales en la vida del individuo. El fundamento de la identidad personal del mexicano reside principalmente en los papeles sociales que desempeñan sus miembros y en la pertenencia a grupos (Sánchez-Aragón y Díaz-Loving, 2009). Sin embargo, cabe señalar que debido a su historia, la desconfianza es un sentimiento que prevalece en los mexicanos: muchas veces prefieren no confiar a sentirse defraudados (Bohórquez, 2010).

En su relación con la autoridad, el mexicano se adapta bien a las reglas e instituciones. Existe una distancia marcada entre los subordinados de las autoridades, y es frecuente que se muestre respeto y formalidad a los individuos de mayor estatus (Díaz-Guerrero, 2003; Sánchez- Aragón y Díaz-Loving, 2009).

Al enfrentar conflictos los mexicanos tienen capacidad de reflexionar sobre la situación y analizar soluciones; sin embargo, dar el paso a la acción no es fácil para ellos (Sánchez-Aragón y Díaz-Loving, 2009). Se dice que son buenos para criticar cuando algo no funciona bien, pero muchos no participan activamente en lograr cambio en la comunidad (Bohórquez, 2010).

La experiencia emocional de los mexicanos se destaca por ser externa e interactiva, favorece la expresividad y el afecto, mientras que limita la expresión de emociones que pueden ser valoradas socialmente como inadecuadas, como enojo o tristeza (Sánchez-Aragón \& Díaz-Loving, 2009). Existen diferencias de género en la expresión de emociones: la mujer es más afectuosa y sensible, mientras el hombre se define por ser más instrumental y enfocado al logro. Los resultados de un estudio realizado por Garay-Hernández, Díaz-Loving, Frías-Cárdenas, Limón-González, Lozano-Verduzco, Rocha-Sánchez, y Zacarías-Guerra (2009) sobre los intereses y actividades de universitarios mexicanos apoyan esta idea, muestran que las mujeres universitarias se centran más en la familia, después en viajar e ir al cine; mientras que los hombres universitarios se enfocan más en su profesión, sus amigos y su familia (Garay-Hernández et al., 2009).

En general, la cultura mexicana se distingue por rasgos de generosidad, valor hacia la unión familiar, y espiritualidad
(Díaz-Guerrero, 2003). Se destaca por ser animada, feliz y optimista, sonreír y compartir son expresiones sociales típicas en los mexicanos (Díaz-Loving, 2005). Tomar en cuenta el aspecto cultural dentro del estudio de las fortalezas de carácter en los individuos es fundamental.

El presente estudio tiene como objetivo describir la percepción de las propias fortalezas, según el modelo de Peterson \& Seligman (2004) en población mexicana, e identificar los puntos convergentes con los modelos ya mencionados.

\section{Método}

La muestra está constituida por 608 personas entre 15 y 82 años de edad, con una media de 28,6 años. Todos residentes del estado de Jalisco y de los cuales el $31 \%$ eran hombres y el $69 \%$ mujeres.

El $32 \%$ es población universitaria, mientras que el $68 \%$ son personas asistentes a las distintas acciones de prevención de la salud mental que ofrece el Departamento de Clínicas de Salud Mental. El 6\% de la muestra alcanzó solamente estudios de primaria, el 7,5\% finalizó la secundaria, el 55\% terminó el bachillerato, el 6\% accedió a una carrera técnica, el 24,5\% estudió licenciatura y el 1\% cuenta con estudios de posgrado.

Instrumentos:

Cuestionario de características sociodemográficas: identifica edad, escolaridad y ocupación. Inventario de Virtudes y Fortalezas (IVyF: Cosentino y Castro, 2008). Se trata de un inventario desarrollado para evaluar las 6 virtudes y 24 fortalezas del carácter según la clasificación de Peterson \& Seligman (2004), incluye 24 ítems bipolares con cinco opciones de respuesta tipo Likert, en la que los encuestados indican en qué grado se parecen más a dos auto descripciones opuestas en función de cada una de las fortalezas. El instrumento ha mostrado una adecuada confiabilidad evaluada a través de la estabilidad test-retest, donde $r$ para cada uno de los reactivos oscila entre .73 y .92. Asimismo el Alfa de Cronbach de la escala total fue de .85 , con una varianza total de 45,27\% (Cosentino, 2009). En este estudio, la puntuación de la confiabilidad interna fue de $\alpha=.846$. Como ejemplo de los reactivos incluimos la descripción de la fortaleza de Creatividad:

Persona 1: Se me ocurren nuevas formas de hacer las cosas porque tengo una mente creativa. Mi imaginación va más allá de la de mis amigos y siento un impulso especial por hacer cosas originales. Así que puedo decir que me gusta ponerme a pensar en ideas nuevas y diferentes que me surgen.

Persona 2: No se me ocurren nuevas formas de hacer las cosas porque no tengo una mente creativa. Mi 
imaginación no va más allá de la de mis amigos ni siento ningún impulso especial por hacer cosas originales. Así que no puedo decir que me guste ponerme a pensar en ideas nuevas y diferentes que me surgen.

La puntuación va de 1 (soy muy parecido a la primera persona) a 5 (soy muy parecido a la segunda persona).

\section{Resultados}

La Tabla 2 muestra los resultados descriptivos en orden ascendente para cada uno de los ítems que conforman el inventario. Se observa que las tres fortalezas menos auto percibidas por la población fueron la autorregulación, el amor por el conocimiento y la modestia; mientras que las tres más altas son gratitud, bondad e integridad.

Tabla 2. Descriptivos para cada una de las fortalezas

\begin{tabular}{|c|c|c|c|c|}
\hline Fortaleza & Media & D.T. & Mínimo & Máximo \\
\hline Autorregulación & 3.08 & 1.12 & 1 & 5 \\
\hline Amor por el conocimiento & 3.41 & .99 & 1 & 5 \\
\hline Modestia & 3.43 & .97 & 1 & 5 \\
\hline Ciudadanía & 3.49 & .99 & 1 & 5 \\
\hline Humor & 3.51 & .99 & 1 & 5 \\
\hline Clemencia & 3.56 & 1.00 & 1 & 5 \\
\hline Espiritualidad & 3.66 & 1.16 & 1 & 5 \\
\hline Esperanza & 3.70 & 1.07 & 1 & 5 \\
\hline Valentía & 3.72 & .96 & 1 & 5 \\
\hline Amor & 3.76 & 1.10 & 1 & 5 \\
\hline Persistencia & 3.79 & 1.05 & 1 & 5 \\
\hline Prudencia & 3.84 & .91 & 1 & 5 \\
\hline Liderazgo & 3.86 & 1.00 & 1 & 5 \\
\hline Inteligencia Social & 3.89 & 1.01 & 1 & 5 \\
\hline Creatividad & 3.89 & .95 & 1 & 5 \\
\hline Apreciación & 3.90 & .97 & 1 & 5 \\
\hline Vitalidad & 3.91 & .88 & 1 & 5 \\
\hline Apertura Mental & 3.95 & .97 & 1 & 5 \\
\hline Curiosidad & 4.04 & .90 & 1 & 5 \\
\hline Imparcialidad & 4.04 & .96 & 1 & 5 \\
\hline Perspectiva & 4.07 & .83 & 1 & 5 \\
\hline Gratitud & 4.13 & .94 & 1 & 5 \\
\hline Bondad & 4.14 & .83 & 1 & 5 \\
\hline Integridad & 4.15 & .89 & 1 & 5 \\
\hline
\end{tabular}


Ante la posibilidad de mostrar las características de las respuestas para cada una de las virtudes, estas fueron agrupándose. En las siguientes tablas (3 a 8), se muestra la distribución de las respuestas en porcentajes por cada una de las fortalezas que integran cada virtud.
Cabe destacar que a excepción del amor del aprendizaje, la ciudadanía, la modestia y la autorregulación, cuyo mayor porcentaje se ubicó en el intermedio, la mayoría de las respuestas se ubicaron con más similitud a la fortaleza que a las características antagónicas.

Tabla 3. Distribución de las respuestas (\%) para las fortalezas que componen la virtud de sabiduría

\begin{tabular}{lccccc}
\hline \multicolumn{1}{c}{ Sabiduría } & $\begin{array}{c}\text { Muy parecido a } \\
\text { la descripción del } \\
\text { antagónico }\end{array}$ & $\begin{array}{c}\text { Algo parecido a } \\
\text { la descripción del } \\
\text { antagónico }\end{array}$ & Entre ambos & $\begin{array}{c}\text { Algo parecido a la } \\
\text { descripción de la } \\
\text { fortaleza }\end{array}$ & $\begin{array}{c}\text { Muy parecido a la } \\
\text { descripción de la } \\
\text { fortaleza }\end{array}$ \\
\hline Creatividad & 1.0 & 7.2 & 23.0 & 38.8 & 29.9 \\
Apertura Mental & 1.0 & 8.4 & 19.1 & 38.2 & 33.4 \\
Curiosidad & 1.0 & 5.3 & 16.8 & 42.9 & 34.0 \\
$\begin{array}{l}\text { Perspectiva } \\
\text { Amor por }\end{array}$ & .7 & 3.8 & 16.1 & 46.9 & 32.6 \\
el aprendizaje & 3.7 & 12.6 & 36.0 & 34.8 & 12.9 \\
\hline
\end{tabular}

Tabla 4. Distribución de las respuestas (\%) para las fortalezas que componen la virtud de coraje

\begin{tabular}{lccccc}
\hline Coraje & $\begin{array}{c}\text { Muy parecido a } \\
\text { la descripción del } \\
\text { antagónico }\end{array}$ & $\begin{array}{c}\text { Algo parecido a } \\
\text { la descripción del } \\
\text { antagónico }\end{array}$ & Entre ambos & $\begin{array}{c}\text { Algo parecido a la } \\
\text { descripción de la } \\
\text { fortaleza }\end{array}$ & $\begin{array}{c}\text { Muy parecido a la } \\
\text { descripción de la } \\
\text { fortaleza }\end{array}$ \\
\hline Valentía & 2.1 & 8.9 & 24.3 & 44.1 & 20.6 \\
Persistencia & 2.3 & 10.7 & 22.2 & 35.5 & 29.3 \\
Integridad & 1.2 & 4.4 & 12.8 & 41.1 & 40.5 \\
Vitalidad & .6 & 5.0 & 24.9 & 42.1 & 27.5 \\
\hline
\end{tabular}

Tabla 5. Distribución de las respuestas (\%) para las fortalezas que componen la virtud de Humanidad

\begin{tabular}{lccccc}
\hline Humanidad & $\begin{array}{c}\text { Muy parecido a } \\
\text { la descripción del } \\
\text { antagónico }\end{array}$ & $\begin{array}{c}\text { Algo parecido a } \\
\text { la descripción del } \\
\text { antagónico }\end{array}$ & Entre ambos & $\begin{array}{c}\text { Algo parecido a la } \\
\text { descripción de la } \\
\text { fortaleza }\end{array}$ & $\begin{array}{c}\text { Muy parecido a la } \\
\text { descripción de la } \\
\text { fortaleza }\end{array}$ \\
\hline Amor & 4.4 & 7.9 & 24.8 & 32.7 & 30.1 \\
Bondad & .7 & 3.3 & 15.0 & 43.6 & 37.5 \\
Inteligencia Social & 1.3 & 9.4 & 20.6 & 36.3 & 32.4 \\
\hline
\end{tabular}


Tabla 6. Distribución de las respuestas (\%) para las fortalezas que componen la virtud de Justicia

\begin{tabular}{cccccc}
\hline Justicia & $\begin{array}{c}\text { Muy parecido a } \\
\text { la descripción del } \\
\text { antagónico }\end{array}$ & $\begin{array}{c}\text { Algo parecido a } \\
\text { la descripción del } \\
\text { antagónico }\end{array}$ & Entre ambos & $\begin{array}{c}\text { Algo parecido a la } \\
\text { descripción de la } \\
\text { fortaleza }\end{array}$ & $\begin{array}{c}\text { Muy parecido a la } \\
\text { descripción de la } \\
\text { fortaleza }\end{array}$ \\
\hline Ciudadanía & 3.1 & 10.9 & 35.4 & 34.9 & 15.8 \\
Imparcialidad & 1.5 & 5.9 & 17.8 & 36.7 & 38.2 \\
Liderazgo & 3.0 & 6.9 & 20.1 & 41.6 & 28.5 \\
\hline
\end{tabular}

Tabla 7. Distribución de las respuestas (\%) para las fortalezas que componen la virtud de Templanza

\begin{tabular}{cccccc}
\hline Templanza & $\begin{array}{c}\text { Muy parecido a } \\
\text { la descripción del } \\
\text { antagónico }\end{array}$ & $\begin{array}{c}\text { Algo parecido a } \\
\text { la descripción del } \\
\text { antagónico }\end{array}$ & Entre ambos & $\begin{array}{c}\text { Algo parecido a la } \\
\text { descripción de la } \\
\text { fortaleza }\end{array}$ & $\begin{array}{c}\text { Muy parecido a la } \\
\text { descripción de la } \\
\text { fortaleza }\end{array}$ \\
\hline Clemencia & 2.8 & 10.5 & 33.1 & 35.4 & 18.3 \\
Modestia & 2.4 & 12.2 & 40.3 & 30.1 & 15.0 \\
Prudencia & 1.5 & 5.4 & 25.3 & 43.1 & 24.7 \\
Autorregulación & 9.0 & 21.2 & 33.6 & 25.3 & 10.9 \\
\hline
\end{tabular}

Tabla 8. Distribución de las respuestas (\%) para las fortalezas que componen la virtud de Trascendencia

\begin{tabular}{cccccc}
\hline Trascendencia & $\begin{array}{c}\text { Muy parecido a } \\
\text { la descripción del } \\
\text { antagónico }\end{array}$ & $\begin{array}{c}\text { Algo parecido a } \\
\text { la descripción del } \\
\text { antagónico }\end{array}$ & Entre ambos & $\begin{array}{c}\text { Algo parecido a la } \\
\text { descripción de la } \\
\text { fortaleza }\end{array}$ & $\begin{array}{c}\text { Muy parecido a la } \\
\text { descripción de la } \\
\text { fortaleza }\end{array}$ \\
\hline Apreciación & 1.2 & 7.4 & 22.9 & 37.3 & 31.3 \\
Gratitud & 1.6 & 4.3 & 16.1 & 35.7 & 42.3 \\
Esperanza & 4.3 & 8.7 & 25.0 & 37.2 & 24.8 \\
Humor & 3.7 & 10.5 & 31.5 & 39.5 & 14.8 \\
Espiritualidad & 6.4 & 8.9 & 24.2 & 33.1 & 27.5 \\
\hline
\end{tabular}

Finamente y con la intención de identificar probables diferencias de género, se realizó un análisis de la varianza, cuyos resultados se encuentran en la Tabla 8, donde destaca que los hombres suelen percibirse con mayor creatividad, mientras que las mujeres, puntúan más alto en persistencia, integridad, amor, bondad, imparcialidad, apreciación por la belleza, buen humor y espiritualidad. 


\begin{tabular}{|c|c|c|c|c|}
\hline Fortaleza & Hombres & Mujeres & $\mathbf{F}$ & Sig. \\
\hline \multicolumn{5}{|l|}{ Sabiduría } \\
\hline Creatividad & 4.05 & 3.83 & 7.530 & .006 \\
\hline Curiosidad & 4.03 & 4.05 & .028 & .867 \\
\hline Apertura Mental & 3.89 & 3.97 & .784 & .376 \\
\hline Amor por el aprendizaje & 3.32 & 3.45 & 1.841 & .175 \\
\hline Perspectiva & 4.10 & 4.06 & .249 & .618 \\
\hline \multicolumn{5}{|l|}{ Coraje } \\
\hline Valentía & 3.76 & 3.70 & .557 & .456 \\
\hline Persistencia & 3.63 & 3.86 & 6.121 & .014 \\
\hline Integridad & 4.02 & 4.21 & 6.208 & .013 \\
\hline Vitalidad & 3.84 & 3.94 & 1.545 & .214 \\
\hline \multicolumn{5}{|l|}{ Humanidad } \\
\hline Amor & 3.46 & 3.89 & 20.827 & .000 \\
\hline Bondad & 3.98 & 4.21 & 10.311 & .001 \\
\hline Inteligencia Social & 3.89 & 3.89 & .007 & .934 \\
\hline \multicolumn{5}{|l|}{ Justicia } \\
\hline Ciudadanía & 3.45 & 3.51 & .384 & .536 \\
\hline Imparcialidad & 3.87 & 4.12 & 8.695 & .003 \\
\hline Liderazgo & 3.90 & 3.84 & .597 & .440 \\
\hline \multicolumn{5}{|l|}{ Templanza } \\
\hline Clemencia & 3.47 & 3.60 & 2.513 & .113 \\
\hline Modestia & 3.41 & 3.44 & .150 & .698 \\
\hline Prudencia & 3.76 & 3.88 & 2.316 & .129 \\
\hline Autorregulación & 3.11 & 3.07 & .165 & .685 \\
\hline \multicolumn{5}{|l|}{ Trascendencia } \\
\hline Apreciación & 3.74 & 3.97 & 7.426 & .007 \\
\hline Gratitud & 4.01 & 4.18 & 4.259 & .039 \\
\hline Esperanza & 3.61 & 3.73 & 1.504 & .221 \\
\hline Humor & 3.36 & 3.58 & 5.758 & .017 \\
\hline Espiritualidad & 3.42 & 3.78 & 12.913 & .000 \\
\hline
\end{tabular}




\section{Discusión y conclusiones}

El presente trabajo presenta una descripción de la percepción de las propias fortalezas según el modelo de Peterson y Seligman (2004) en población mexicana. Los resultados plantean que las principales fortalezas auto percibidas son integridad, bondad, gratitud, perspectiva e imparcialidad. Las menos percibidas son auto regulación, amor por el aprendizaje, modestia, ciudadanía y humor.

Contrastando con el estudio transcultural llevado a cabo por Park, Peterson \& Seligman (2006), nuestros resultados coinciden solo con cuatro de las fortalezas: integridad y gratitud entre las que obtienen mayores puntuaciones, $y$ autorregulación y modestia entre las menos percibidas tanto a nivel internacional como entre la población mexicana. Cabe señalar así mismo que nuestros resultados difieren de los encontrados en la muestra mexicana en el estudio realizado por Park et al. (2006), no obstante la muestra referida en dicho estudio fue de solo 88 participantes, con escolaridad universitaria y bilingües, por lo que no podemos considerar que sea representativa de las características de los mexicanos; aunque se mantuvieron en la misma tendencia la gratitud, la auto regulación y la modestia. Se observa el mismo fenómeno al contrastar los resultados con el estudio con participantes españoles (Azañedo et al., 2014).

Destacan también las diferencias culturales con población inglesa, donde el amor por el conocimiento se encuentra entre las más altas, pero nuevamente la auto regulación y la modestia se observan entre las puntuaciones más bajas (Linley et al., 2007).

En un intento de identificar los posibles puntos de convergencia entre estos modelos y los estudios de las dimensiones culturales, y desde la perspectiva de la psicología social en México, también encontramos similitudes. Por ejemplo Hofstede et al. (2010) y Díaz-Loving y Draguns (1999), identifican un perfil cultural nacional para México, que se caracteriza principalmente por ser colectivista y poner el bienestar común por encima del personal, coincide con las altas puntuaciones obtenidas en las fortalezas de bondad, gratitud y perspectiva, precisamente asociadas a la vinculación interpersonal. Así mismo, las puntuaciones elevadas en la dimensión de masculinidad, pueden estar relacionadas con la baja auto regulación, como sucede para las culturas inglesa y española que presentan puntaciones similares en este rubro (Hofstede et al., 2010; Azañedo et al., 2014 y Linley et al., 2007). Por otro lado, esta falta de auto regulación, coincide con la caracterización de la experiencia emocional de los mexicanos, que se destaca según Sánchez-Aragón \& Díaz-Loving (2009) por ser externa e interactiva; no obstante, este último trabajo considera que también suele limitarse la expresión de emociones que son vistas como inadecuadas.

Entre los puntos que consideramos discordantes entre nuestros resultados y los estudios de las dimensiones culturales, destaca la baja puntuación en humor, ya que esta fortaleza es característica de los mexicanos (Díaz-Loving, 2005) y pudiera explicar el alto índice de indulgencia, así como parte esencial del colectivismo, por lo que se sugiere como prospectiva de investigación ahondar más en esta posible relación, en su forma de evaluar la dimensión de buen humor y del efecto de deseabilidad social.

Surge la pregunta sobre si las diferencias entre los indicadores de fortalezas y características de las dimensiones culturales podrían relacionarse con los indicadores de bienestar social y desarrollo económico de los países; es decir, si la prevalencia de determinadas fortalezas como parte de la identidad nacional puede impactar en indicadores macro sociales.

Respecto a las diferencias de género encontradas en las fortalezas humanas evaluadas en este estudio, también coinciden con los resultados obtenidos en la muestra española, donde los hombres puntuaron más alto en creatividad (Azañedo, et al., 2014; Linley et al., 2007); mientras que las mujeres en amor, bondad y gratitud (Azañedo et al., 2014). En nuestro estudio se observaron diferencias: mayores puntuaciones para las mujeres en persistencia, integridad, apreciación por la belleza, humor, espiritualidad e imparcialidad.

Corroborar las coincidencias entre los modelos, pero sobre todo identificar un perfil de población mexicana respecto a la percepción de las propias virtudes, resulta útil para la generación de programas de formación en dichas fortalezas y en su aplicación en el entorno cotidiano, principalmente en lo que se refiere a la re-estructuración del tejido social a través de una convivencia en armonía y con el fin de aportar a la solución de muchos de los problemas sociales en el contexto de México.

Cabe señalar como limitante de este estudio que la muestra, aunque heterogénea en cuanto edad y niveles socioeducativos, se constituyó solamente con personas residentes en el estado de Jalisco, por lo que es deseable indagar estas mismas características en una población representativa de todos los estados del país. 


\section{Referencias}

Azañedo, C.M., Fernández-Abascal, E.G., \& Barraca, J. (2014). Character strengths in Spain: Validation of the Values in Action Inventory of Strengths (VIA-S) in a Spanish sample. Clínica y Salud, 25 (2), 123-130.

Bok, S. (1995). Common values. Columbia, MO: University of Missouri Press. Bohórquez, E. (2010). La psicología del mexicano. Revista Hoy y Ahora, 42-49.

Castro, J. (2011). Las rutas de acceso al bienestar. Relaciones entre bienestar hedónico y eudaemónico. Un estudio con población Argentina. RIDEP, 31 (1), 37-55.

Cosentino, A. (2009). Evaluación de las virtudes y fortalezas humanas en población de habla hispana. Psicodebate, Psicología, Cultura y Sociedad, 10, 53-71.

Cosentino A., y Castro A. (2008). Inventario de virtudes y fortalezas. Manuscrito no publicado.

Corral-Verdugo, V., Tapia-Fonllem, C., Ortiz-Valdez, A., y Fraijo-Sing, B. (2013). Las virtudes de la humanidad, justicia y su relación con la conducta sustentable. Revista Latinoamericana de Psicología, 45 (3), 363-374.

Díaz-Loving, R., \& Draguns, J. (1999). Socioculture, meaning, and personality in México and the United States. En Y.T. Lee, C. McCaulloy, \& J. Draguns, J. (Eds.), Personality and Perception Across Cultures. London, UK: Lawrence Erlbaum Associates.

Díaz-Guerrero, R. (2003). La Psicología del Mexicano. México, D.F.: Editorial Trillas.

Díaz- Loving, R. (2005). Emergence and contributions of a Latinamerican indigenous social psychology. International Journal of Psychology, 40 (4), 213-227.
Garay, J., Díaz-Loving, R., Frías-Cárdenas, M.T., Limón- González, B., Lozano-Verduzco, I., Rocha-Sánchez, T.E., y Zacarías-Guerra, M. (2009). Intereses y valores en jóvenes mexicanos. Enseñanza e Investigación en Psicología, 13(2), 201-214.

Hofstede, G., Hofstede, G.J., \& Minkov, M., (2010). Cultures and organizacións: Software of the mind. New York: McGraw Hill

Linley, P. A., Maltby, J., Wood, A. M., Joseph, S., Harrington, S., Peterson, C., \& Park, N. (2007). Character strengths in the United Kingdom: The VIA Inventory of Strengths. Personality and Individual Differences, 43, 341-351. doi:10.1016/j.paid.2006.12.004

Martínez-Martí, M.L. (2006). El estudio científico de las fortalezas trascendentales desde la psicología positiva. Clínica y Salud, 17 (3), 245-258.

Peterson, C. \& Seligman, M. (2004). Character Strenghts and Virtues. A Handbook and Classification. New York, NY: Oxford University Press.

Peterson, C. \& Park, N. (2005). Classification and measurement of character strengths. Implications for practice (pp. 433-446) In P.A. Linley and S. Joseph (Eds). Positive Psychology in Pratice. New Jersey: John Wiley \& Sons.

Park, N., Peterson, C., \& Seligman, M. (2006). Character strengths in fifty four nations and the fifty US states. Journal of Positive Psychology, 1 (3), 118-129.

Sánchez-Aragón, R., y Díaz-Loving, R. (2009). Las reglas y preceptos culturales de la expresión emocional en México: Su medición. Universitas Psychologia, 3(3), 793-805. 
Anexo 1. Descripción de las fortalezas humanas (Peterson \& Seligman, 2004)

\begin{tabular}{|c|c|}
\hline \multicolumn{2}{|r|}{ SABIDURÍA } \\
\hline Creatividad & Se refiere a la generación de ideas o conductas que sean originales, innovadoras, productivas y adaptativas. \\
\hline Curiosidad & $\begin{array}{l}\text { Es la respuesta activa con propósito y regulación ante los retos y oportunidades que implican aprendizaje, así como el } \\
\text { estar abierto a las experiencias y prestar interés a las experiencias vividas }\end{array}$ \\
\hline Apertura Mental & $\begin{array}{l}\text { Implica la voluntad para identificar evidencias de los hechos, incluso a pesar de las propias creencias, examinar las cosas } \\
\text { desde distintos puntos de vista, así como la posibilidad de cambiar de opinión en caso de que dichas evidencias sean factibles }\end{array}$ \\
\hline Amor por el Saber & $\begin{array}{l}\text { Describe el modo en el que una persona se implica en la obtención de nueva información, en el dominio de nuevas } \\
\text { habilidades, temas y áreas de conocimiento }\end{array}$ \\
\hline Perspectiva & $\begin{array}{l}\text { Esta fortaleza la poseen las personas que se auto conocen, toman decisiones acertadas, son congruentes, comprenden } \\
\text { sus limitaciones, cuentan con un amplio criterio y son capaces de brindar un sabio consejo a los demás }\end{array}$ \\
\hline \multicolumn{2}{|r|}{ CORAJE } \\
\hline Valentía & $\begin{array}{l}\text { Se refiere a la disposición voluntaria para actuar a pesar del temor en circunstancias donde existen riesgos con el objetivo } \\
\text { de preservar u obtener algo considerado como positivo. Implica afrontar las amenazas, los retos, las dificultades y dolores. }\end{array}$ \\
\hline Persistencia & Implica la autodirección para obtener logros y llegar a las metas a pesar de la existencia de obstáculos \\
\hline Integridad & $\begin{array}{l}\text { Implica la aceptación de la responsabilidad por las emociones y conductas propias, así como la honestidad consigo } \\
\text { mismo y presentarse de manera genuina }\end{array}$ \\
\hline Vitalidad & Es la experiencia subjetiva de sentirse con energía, entusiasmo, ser eficaz y mantenerse alerta \\
\hline \multicolumn{2}{|r|}{ HUMANIDAD } \\
\hline Amor & $\begin{array}{l}\text { Representa una postura cognitiva, conductual y emocional que favorece la protección y el cuidado hacia los demás, así } \\
\text { como la valoración de las relaciones cercanas }\end{array}$ \\
\hline Bondad & Es una fortaleza caracterizada por la disposición a ser generoso, amable, compasivo y altruista con los demás \\
\hline Inteligencia Social & $\begin{array}{l}\text { Se refiere a la habilidad para identificar las diferencias y semejanzas con los demás, identificar las dinámicas interpersonales } \\
\text { y generar relaciones sociales constructivas }\end{array}$ \\
\hline \multicolumn{2}{|r|}{ JUSTICIA } \\
\hline Ciudadanía & $\begin{array}{l}\text { Implica un sentido de obligación hacia el bien común y a la conciencia de los límites entre los intereses personales y los } \\
\text { sociales }\end{array}$ \\
\hline Imparcialidad & $\begin{array}{l}\text { Es un producto del juicio moral que determina lo que es correcto o incorrecto. Implica tratar a todos por igual de acuerdo } \\
\text { a las nociones de rectitud y justicia. }\end{array}$ \\
\hline Liderazgo & $\begin{array}{l}\text { Es una cualidad personal que integra atributos cognitivos y temperamentales para dirigir y motivar y supervisar la acción } \\
\text { de los demás. }\end{array}$ \\
\hline
\end{tabular}




\begin{tabular}{|c|c|}
\hline \multicolumn{2}{|r|}{ TEMPLANZA } \\
\hline Clemencia & Se refiere a la disposición para perdonar una vez que se recibió un daño o transgresión. \\
\hline Modestia & Implica la estimación moderada de los méritos y logros. \\
\hline Prudencia & $\begin{array}{l}\text { Es una forma práctica de razonamiento y automanejo encaminado al logro efectivo de metas, a través de responsabilizarse } \\
\text { de las propias acciones y un claro análisis de los costos beneficios de las acciones llevadas a cabo. }\end{array}$ \\
\hline Auto-regulación & $\begin{array}{l}\text { Se refiere a como una persona maneja sus propias respuestas cognitivas, emocionales y conductuales mediado por las } \\
\text { reglas morales, además de las normas y las expectativas sociales }\end{array}$ \\
\hline \multicolumn{2}{|r|}{ TRASCENDENCIA } \\
\hline Apreciación & $\begin{array}{l}\text { Se refiere a la habilidad para encontrar, reconocer, admirar y disfrutar de la existencia en los aspectos tanto físicos como } \\
\text { sociales }\end{array}$ \\
\hline Gratitud & Es el sentido de agradecimiento ante lo recibido, ya sean aspectos físicos, afectivos o sociales \\
\hline Esperanza & Implica pensar en prospectiva y esperar que se cumpla lo deseado \\
\hline Humor & Es la generación de alegría y gozo, incluso en la adversidad y la habilidad de hacer sentir alegres a los demás \\
\hline Espiritualidad & $\begin{array}{l}\text { Se refiere a las creencias y prácticas que rodean la convicción de que existen dimensiones que no son físicas, pero sí } \\
\text { trascendentes y que otorgan sentido a la existencia }\end{array}$ \\
\hline
\end{tabular}

